\title{
Convolutional Neural Networks for Cellular Automata Classification
}

\author{
Eric Silverman \\ MRC/CSO Social and Public Health Sciences Unit, \\ University of Glasgow, Glasgow G2 3AX, UK \\ eric.silverman@glasgow.ac.uk
}

\begin{abstract}
Wolfram famously developed a four-way classification of CA behaviour, with Class IV containing CAs that generate complex, localised structures. However, finding Class IV rules is far from straightforward, and can require extensive, timeconsuming searches. This work presents a Convolutional Neural Network $(\mathrm{CNN})$ that was trained on visual examples of CA behaviour, and learned to classify CA images with a high degree of accuracy. I propose that a refinement of this system could serve as a useful aid to CA research, automatically identifying possible candidates for Class IV behaviour and universality, and significantly reducing the time required to find interesting CA rules.
\end{abstract}

Some Cellular Automata (CAs) are capable of unexpectedly complex behaviour despite being based on simple rules. Wolfram's early investigations into the behaviour of onedimensional Elementary CAs (ECAs) led him to propose a four-way qualitative classification scheme for CAs according to the behaviour they exhibit from random initial conditions (1984): Class I CAs converge to a homogeneous state; Class II CAs generate periodic or stable structures; Class III CAs generate random or chaotic patterns; and Class IV CAs produce complex localised structures.

The CA community has devoted a great deal of effort toward understanding these classes of behaviour, refining their definitions, and cataloguing interesting CA rules (see e.g. $\mathrm{Li}$ et al., 1990). However, determining whether a CA is in Class IV is not only difficult, it is undecidable (Culik and $\mathrm{Yu}, 1988$ ). Nonetheless, classifying CAs in this way, or with related schemes, remains a useful and succinct way to describe the behaviour observed in CAs qualitatively, and identify CAs worthy of further exploration.

Below I demonstrate how a convolutional neural network (CNN) can successfully classify one-dimensional CAs into the four Wolfram classes.

\section{Methods}

CNNs were chosen for this task due to their notable success in computer vision tasks (Krizhevsky et al., 2012). This $\mathrm{CNN}$ takes greyscale images of CA behaviour as input, and outputs the Wolfram class of the image. The first iteration,

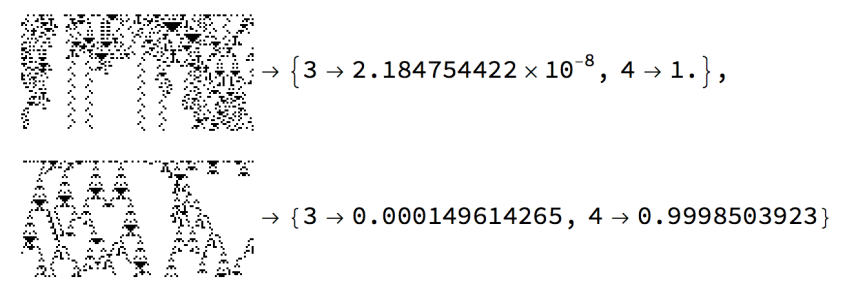

Figure 1: Classification certainty of Network II on novel CA examples. Network II successfully classified $99.8 \%$ of 6,145 novel images in the test set.

Network I, consisted of nine layers: a 16x59x118 convolution layer; 16x59x118 ReLU layer; 16x58x116 convolution layer; 16x58x116 ReLU layer; 16x1x1 pooling layer; 16-node flattening layer; 256 -node fully-connected layer; 4node fully-connected layer; and 4-node SoftMax layer ${ }^{1}$. The network was built in Mathematica 12 and trained on a set of 32,768 120x60-pixel images of the evolution of all 256 elementary CA rules from different random initial conditions. Network I was trained for two hours on four CPU cores, at which point accuracy on the training set had exceeded 99.93\%.

A second CNN with the same structure, Network II, was trained on 3- and 4-colour totalistic CA rules as well as the ECA data, in order to increase the network's exposure to Class IV behaviour. The network was built in Mathematica 12 and trained on a new set of 16,384 ECA examples, plus an additional 8,192 samples drawn from eight known Class IV 3- and 4-colour totalistic rules (codes 1041, 1388, 1635, $1815,2007,2043,2049$, and 1004600). This network was trained for two hours using four CPU cores, at which point accuracy on the training set exceeded $99.2 \%$.

Network III, again using the same structure, was trained on larger images with a resolution of 200 by 120 pixels. The training set included an additional 8,192 images generated

\footnotetext{
${ }^{1}$ This architecture was inspired by a project by Thales Fernandes on identifying ECA rules from images: https:// community.wolfram. com/groups/-/m/t/1417114
} 

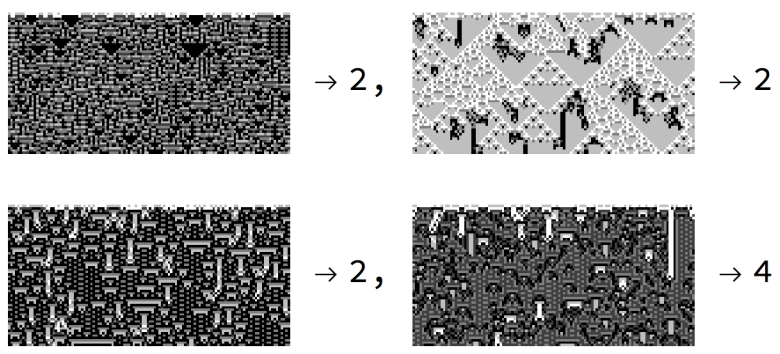

Figure 2: The four highest-entropy inputs to Network II from a test set of $k=5, r=1$ examples.

from $k=5, r=1$ totalistic rules to provide further examples of Class IV behaviour ${ }^{2}$. This network was trained for three hours on four CPU cores, at which point accuracy on the training set exceeded $99.7 \%$.

\section{Results}

Network I achieved an accuracy of $100 \%$ on a test set composed of 4,096 additional ECA and totalistic CA examples (see Figure 1). Testing with images from previously unseen $k=4, r=1$ totalistic CAs produced poor results $(18.6 \%$ accuracy), with Class IV inputs frequently misclassified as Class II/III.

Network II was trained with the larger data set in an effort to improve generalisation. This network correctly classified $99.8 \%$ of a test set of 6,145 novel images of Class IV 3colour totalistic rules; Figure 1 shows some examples. The network was not able to generalise to 5-colour CAs, classifying a set of 1,024 examples with $57.6 \%$ accuracy. Figure 2 shows four of the highest-entropy $k=5, r=1$ test set inputs (where the class determination was the most uncertain). The high-entropy inputs from the test set suggest the most common errors are mistaking visually complicated Class II CAs for III or IV, and Class III CAs for Class IV.

Network III, having been trained on images derived from 5-colour CAs, was $99.9939 \%$ accurate on a 16,384-image test set including 2, 3, 4 and 5-colour CA images; in the entire test set only one image was misclassified (a Class III was mistaken for Class IV). For this network, the highestentropy images from the test set were all Class IV examples, but they were all correctly classified (see Figure 3).

\section{Conclusions and Future Work}

Network II's ability to generalise to new examples of Class IV behaviour in the space of 3-colour and 4-colour totalistic rules suggests that CNNs can be a useful aid in the search

\footnotetext{
${ }^{2}$ These data were generated by rules found through a random search and classified manually. Details of the CA rules and methods used in this project can be found at https://github.com/thorsilver/ Neural-Networks-for-CA-Classification
}
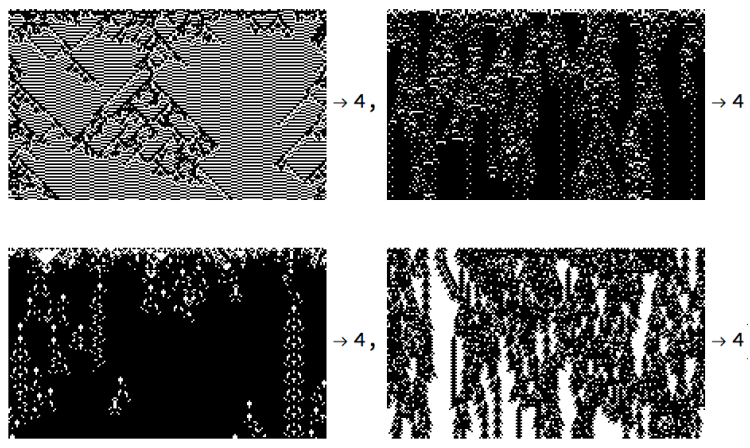

Figure 3: The highest-entropy images in the test set according to Network III. All but one of the images in the 16,384image test set were correctly classified.

for CA complexity. A suitably-trained CNN could identify likely Class IV candidates quickly and significantly reduce the numbers of CA rules that need to be manually checked. This CNN can make classification judgments using only a single visual input, as compared to other methods that may require time-consuming statistical tests.

Network III demonstrates that only a few examples of behaviour in higher-complexity rule spaces are necessary to allow the network to identify Class IV rules in that space. However, success in 6-colour CAs remains elusive; these CAs are nearly universally seen as Class IV by the network due to their increased visual complexity. Work is ongoing with deeper networks trained on larger images, with the goal of producing a network capable of detecting candidate Class IV behaviour even in unexplored CA rule spaces.

\section{Acknowledgements}

Eric Silverman is part of the Complexity in Health Improvement Programme supported by the Medical Research Council (MC_UU_12017/14) and the Chief Scientist Office (SPHSU14). Thanks are due to Stephen Wolfram and Hector Zenil for their valuable comments and advice on this work.

\section{References}

Culik, II, K. and Yu, S. (1988). Undecidability of CA classification schemes. Complex Systems, 2(2):177-190.

Krizhevsky, A., Sutskever, I., and Hinton, G. E. (2012). ImageNet classification with deep convolutional neural networks. In Advances in Neural Information Processing Systems, pages 1097-1105.

Li, W., Packard, N. H., and Langton, C. G. (1990). Transition phenomena in cellular automata rule space. Physica D: Nonlinear Phenomena, 45(1-3):77-94.

Wolfram, S. (1984). Universality and complexity in cellular automata. Physica D: Nonlinear Phenomena, 10(1-2):1-35. 